\title{
ALGUNAS CONSIDERACIONES SOBRE EL INICIO DE LA ARQUERÍA PREHISTÓRICA
}

\author{
SOME THOUGHTS ON THE BEGINNING \\ OF PREHISTORIC ARCHERY
}

FRANCISCO JAVIER MUÑOZ IBÁÑEZ $(*)$

\section{RESUMEN}

En este trabajo se aborda el posible origen de la arquería prehistórica durante el Solutrense Superior en la vertiente mediterránea de la Península Ibérica. A partir del análisis de las puntas ligeras de proyectil del Solutrense Extracantábrico (punta de aletas y pedúnculo y punta de muesca de tipo mediterráneo) se establecen las características balísticas de este tipo de utillaje. Estas características permiten establecer algunas hipótesis sobre su funcionalidad en relación a los sistemas de engaste y propulsión.

\begin{abstract}
This paper deals with the study of the possible origin of prehistoric archery during the Upper Solutrean in Mediterranean Iberia. From analysis of the light arrowheads of the Extracantabrian Solutrean (barbed and tanged point and the shouldered points of Mediterranean type) are established the ballistics characteristic of this type of tool kit. These characteristics permit us to establish some hypothesis on the function in relation to their mounting and propulsion.
\end{abstract}

Palabras clave: Arquería prehistórica. Punta de aletas y pedúnculo. Punta de muesca de tipo mediterráneo. Solutrense.

Key words: Prehistoric archery. Barbed and tanged point. Shouldered point of Mediterranean type. Solutrean.

(*) Departamento de Prehistoria e Historia Antigua de la Universidad Nacional de Educación a Distancia. Avda. Senda del Rey s/n. 28071 Madrid.

El artículo fue remitido en su versión final el 30-III-99.

\section{INTRODUCCIÓN}

La idea generalizada de la funcionalidad como elementos arrojadizos de las puntas solutrenses ha sido casi siempre más intuitiva que científica. Sin embargo, el examen exhaustivo tanto de las puntas de aletas y pedúnculo y como de las puntas de muesca de retoque abrupto del Solutrense Extracantábrico ha demostrado que este tipo de utillaje fue concebido para un uso como punta ligera de proyectil (Muñoz, 1997, e.p.). Este estudio se realizó sobre una muestra de 70 puntas de aletas y pedúnculo y 511 puntas de muesca procedentes de los yacimientos de la Cova del Parpalló (Gandía, Valencia) y la Cueva de Ambrosio (Vélez Blanco, Almería) (Fig. 1). Ambas estaciones son las únicas con series líticas especialmente significativas para realizar un análisis de esta naturaleza. En el resto de las estaciones del Solutrense Extracantábrico, la extrapolación que se podría hacer de sus exiguas colecciones de puntas de proyectil no sería suficientemente representativa de las mismas.

Diversos autores (Jordá, 1958; Smith, 1966; Rasilla, 1989) han especulado sobre los sistemas de propulsión del utillaje solutrense. Entre ellos, como idea más recurrente se sitúa el posible uso del arco como una invención de este periodo. Incluso, diversos especialistas en arquería prehistórica y antigua propugnan la posible aparición del arco en el Paleolítico Superior Inicial, con las puntas de la FontRobert como los primeros proyectiles de flecha, (Bergman et alii, 1988).

Sin embargo, su evidencia material no se documenta hasta el Mesolítico. Esto ha llevado a otros autores (Rozoy, 1978, 1992, 1993) a situar el origen de la arquería en este momento, considerando los 


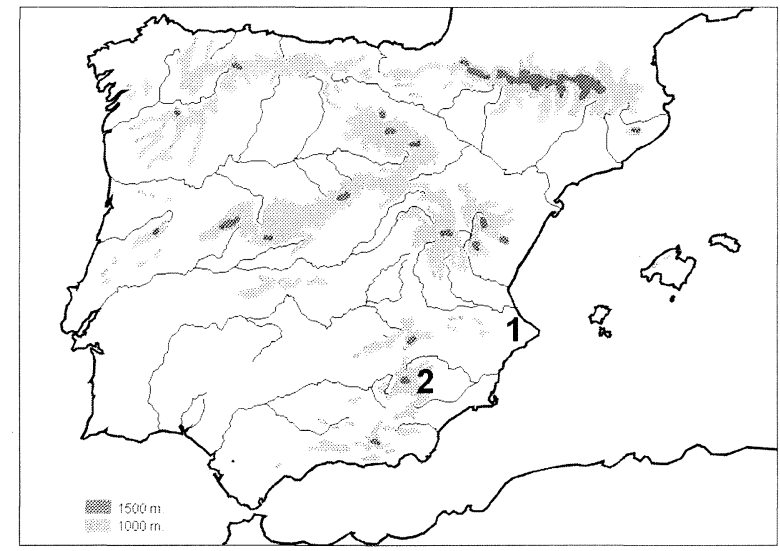

Fig. 1. Mapa de situación de los yacimientos estudiados: 1. Cova del Parpalló (Gandía, Valencia); 2. Cueva de Ambrosio (Vélez Blanco, Almería).

elementos microlíticos como las primeras puntas de flecha. Es indudable que los primeros testimonios de arcos, astiles, puntas enmangadas en éstos y restos óseos con marcas de penetración de proyectiles, incluso puntas clavadas sobre ellos, se sitúan en este periodo.

Los primeros arcos documentados aparecen en el norte y este de Europa, donde las características singulares del depósito arqueológico han permitido su conservación. En muchos casos se trata de evidencias recuperadas en zonas pantanosas, hábitat en palafitos, o en regiones de tundra, donde se han creado las condiciones necesarias para que hayan podido llegar relativamente intactos hasta nuestros días. Los ejemplares más antiguos corresponden a los arcos recuperados en el yacimiento danés de Elm (Alrune, 1992), que está fechado en el Dryas III, y en el alemán de Stellmoor (Rust, 1943), datado en el mismo periodo. Siguiendo una evolución cronológica, en el final del Boreal se situarían la estación danesa de Holmegaard (Becker, 1945; Mathiassen, 1948) y en la cuenca del Petchora el yacimiento ruso de Wis (Bourov, 1973), cuya cronología oscila entre el final del Boreal y el inicio del Atlántico. Asimismo, del final de este periodo, el Ertebölliense, como últimos ejemplares mesolíticos se documentan los arcos aparecidos en las estaciones danesas de Muldbjerg (Troels-Smith, 1959) y Braband (Tomsen y Jessen, 1904). También, del Neolítico se han podido recuperar algunos arcos, como el del yacimiento ingles de Meare Heart (Clark, 1963) o el de Charavines en Francia (Bocquet, 1994), datados en el 2960 \pm 120 a.C. y hacia el 5000 a.C., respectivamente.
Como en el caso de los arcos, las primeras evidencias de astiles de flecha conservados pertenecen a estaciones situadas en latitudes septentrionales. En el yacimiento alemán de Stellmoor (Rust, 1943), cerca de Hamburgo, se han recuperado casi un centenar de astiles con una ranura estrecha y rectangular en su extremo proximal para ser encajada en la cuerda del arco, que se fechan en el Dryas III. En la estación danesa de Loshult (Petersson, 1951; Malmer, 1968) se encontraron dos ejemplares pertenecientes al Boreal Antiguo. Asimismo, en Vinkel se documentan varios astiles datados en la fase final del Boreal (Troels-Smith, 1961), igual que los del yacimiento ruso de Wis (Bourov, 1973).

\section{EL ARCO}

El concepto de arco es, en principio, muy simple: dos brazos armados por medio de una cuerda que los mantiene en tensión, (Hamilton, 1982). En arquería se entiende por tensión la longitud a la cual puede tensarse un arco. Esta tensión almacena en el arco una energía potencial que se transfiere de la cuerda a la flecha cuando ésta se dispara. $\mathrm{La}$ energía potencial es producto de la fuerza de tracción que se genera a lo largo del lomo o curva exterior y de la fuerza de compresión que se desarrolla en el vientre o curva interior. Cualquier arco debe adaptarse a estas fuerzas a fin de evitar que se rompa. Los arcos antiguos se clasifican en tres categorías en función de los materiales usados en su construcción (Bergman et alii , 1988):

- Arco simple: realizado a partir de una sola pieza de madera.

- Arco reforzado: es un arco simple con un tendón engomado en el dorso que permite aumentar la velocidad y distancia de tiro, al mismo tiempo que se reduce el riesgo de fractura al necesitar una menor tensión.

- Arco compuesto: consta de un fino núcleo de madera en el que se pega un tendón en el lomo y cuerno en el vientre. La combinación de estos materiales permite soportar al arco una gran fuerza de tracción y compresión, con un riesgo casi nulo de fractura aunque los brazos sean muy cortos.

Todos los arcos conocidos, tanto del Mesolítico como del Neolítico, se corresponden con el primer tipo. Aunque algunos autores (Rozoy, 1978) argumentan la posibilidad de la existencia de tipos reforzados con tendones, el registro arqueológico no indica la presencia de los mismos. En su inmensa 
mayoría están realizados en tejo (Taxus baccata), éste sería el caso del ejemplar de Meare Heath, y en olmo (Ulmus sp.) como los de Holmegaard y Muldbjerg. No obstante, cualquier madera que sea relativamente elástica para resistir las fuerzas de tensión y compresión sin quebrarse y tenga la capacidad de recuperar su forma original después del esfuerzo, pudo ser usada en la fabricación de estos arcos primitivos. Así, el encontrado en Braband estaba realizado en fresno (Fraxinus excelsior), e incluso los arcos de Wis eran de conífera (Picea abies y Pinus sylvestris). Aún así, siempre el tejo es la primera materia prima elegida en la construcción de arcos y solo cuando no está disponible se opta por otras especies arbóreas.

Además, la madera escogida no debe tener en su interior ni nudos, ni granos retorcidos, ya que esto provoca una respuesta diferencial a la tensión en las distintas partes del arco, lo que acaba por deformarlo o fracturarlo. Por último, también hay que tener en cuenta la combinación ideal de duramen y de albura. Cuando se tensa el arco, la albura, que forma el lomo, también se dobla mientras que el duramen, que forma el vientre, se contrae. La flexibilidad de la albura impide que el duramen, que forma el núcleo central, se fracture. Si la proporción de albura es demasiado alta, el arco no recupera su forma original y, a la inversa, éste no posee la suficiente elasticidad para aguantar la tensión. Todos estos factores condicionan que los troncos o ramas de los que es posible construir arcos de una sola pieza sean muy escasos.

Los primeros arcos mesolíticos presentan unos brazos anchos y aplanados, más delgados y estrechos en los extremos que en el centro. Asimismo, la parte interior está finamente trabajada, dándole al arco una sección semicircular o hemilenticular. El lomo redondeado y el vientre plano aprovechan en gran medida la morfología original de la rama o árbol seleccionado. Además, esta forma es la que menos alteración provoca en las distintas capas de albura, lo que evita un debilitamiento de la materia prima al no trabajar la madera a contraveta. De lo contrario, las fibras leñosas se separan y el arco se fisura. El ejemplar de Holmegaard presenta una empuñadura más estrecha y gruesa, lo que facilita el tiro y aumenta la tensión sin disminuir la resistencia. Uno de los arcos del yacimiento de Wis presenta una gran curvatura cuando no está armado, muy probablemente debido al tipo de materia prima empleado que es poco flexible y mediante este diseño se reducen las posibilidades de fractura, consiguiéndose una mayor longitud de lanzamiento con una menor tensión.

Los arcos simples tienen palas bastante largas para obtener una tensión importante sin que el arco se doble excesivamente y con ello se disminuya el riesgo de fractura, con valores asimilables a la arquería medieval. Esta longitud de brazos permite efectuar un tiro rápido y de largo alcance. Los arcos de Holmegard oscilan entre $150 \mathrm{~cm}$. y $180 \mathrm{~cm}$., el de Muldbjerg mide $170 \mathrm{~cm}$., los ejemplares de Wis varían entre $130 \mathrm{~cm}$. y $150 \mathrm{~cm}$., aunque aquí hay un ejemplar desmesuradamente largo que alcanza los $3,5 \mathrm{~m}$.

\section{LA FLECHA}

La flecha está formada por el astil -emplumado o no en su extremo proximal-y la punta de proyectil en el extremo distal. Presentan un tamaño y peso variable en función de la longitud de tensión del arco, así como de la propia función para la que fue ideada la flecha. No es igual una flecha concebida para matar un ave y obtener sus plumas que otra destinada a cazar un búfalo a caballo.

La afirmación según la cual, un arquero de estatura media no puede disparar una flecha de más de $75 \mathrm{~cm}$. porque es la longitud disponible aproximada entre el brazo tenso que sujeta el arco y el brazo flexionado que tira de la cuerda (Pope, 1962), no es del todo correcta ya que la punta de flecha no necesariamente tiene que llegar hasta el lomo del arco. Por ejemplo, algunas flechas de caña de los indios brasileños son únicamente tensadas hasta $1 / 3$ de su longitud total, (Heath y Chiara, 1977). Por regla general, en los arcos simples las flechas no pueden tensarse más de la mitad de su longitud total sin que haya un riesgo importante de fractura, (Hamilton, 1982). Por lo tanto, la relación entre la longitud del arco y la longitud de tensión no puede ser mayor de $2: 1$. Sin embargo, en las experimentaciones de $\mathrm{C}$. Bergman, E. McEven y R. Miller (1988) con un arco simple de tejo, esta relación llega a 2,4:1. La longitud de los ejemplares arqueológicos presenta muy pocas variaciones. En Homelgaard oscilan entre los $70 \mathrm{~cm}$. y los $90 \mathrm{~cm}$. Aquí los astiles más cortos presentan dos muescas en " $V$ " que permiten unir dos piezas en una sola hasta alcanzar longitudes máximas de casi $1 \mathrm{~m}$. La parte más corta es donde se aloja la punta. Al ser esta zona la más frecuentemente fracturada, este sistema permite reemplazar sólo el fragmento dañado y no hacer un as- 
til totalmente nuevo, que necesita una gran inversión de tiempo en su fabricación. En Loshult el ejemplar conservado alcanza los $88 \mathrm{~cm}$., mientras que en Vinkel se sitúan entre $101 \mathrm{~cm}$. y $102 \mathrm{~cm}$. El diámetro de los astiles oscila, por regla general, entre los $8 \mathrm{~mm}$. y los $9 \mathrm{~mm}$., siendo muy raros los ejemplares que sobrepasan los $10 \mathrm{~mm}$. y los que no alcanzan los $5 \mathrm{~mm}$. Por lo tanto, la evidencia arqueológica constata que la relación entre la longitud de tensión y la longitud de la flecha es algo superior a $2: 1$. No obstante, es imposible conocer si la tensión de las palas provocaba que la punta de proyectil retrocediera hasta el lomo o por el contrario no se tensara hasta su longitud total.

El material para la fabricación de los astiles puede ser muy variado y su elección depende en gran medida de los recursos que ofrezca el entorno. Árboles jóvenes, ramas y cañas son un buen soporte para la obtención de astiles. En todos los yacimientos citados la madera usada es el pino (Pinus sylvestris) y en todos los casos los astiles se han elaborado a partir de troncos muy gruesos, eliminando toda la albura y dejando únicamente el núcleo central del duramen. La estimación que hace A. Rust (1943) sobre la edad de los árboles usados en la fabricación de los astiles de Stelmoor, 50 años, parece excesivamente elevada. Lo que sí queda constatado es que las flechas se realizan a partir de árboles relativamente viejos y no sobre ramas jóvenes. Los troncos están cuidadosamente elegidos y no presentan nudos o deformaciones. A pesar de esta esmerada selección, es probable que algunos ejemplares debieran ser rectificados por calentamiento para conseguir una morfología rectilínea, debido a que el uso de cinceles de hueso para la extracción de los soportes (Rozoy, 1978) no permite una total precisión en este trabajo. Incluso algunos investigadores (Olsen, 1973; Bergman et alii, 1988) han indicado que el bastón de mando podría servir para este uso. Sin embargo, los astiles de madera enderezados por calor, generalmente, permanecen menos tiempo rectos. Los astiles han sido cuidadosamente pulidos mediante raspado y/o abrasión, aunque es muy difícil individualizar una técnica de otra ya que sus resultados son muy parejos (Stordeur-Yedid, 1975; Camps-Fabrer, 1975).

El uso de otras especies vegetales, como la caña, es actualmente muy difícil de atestiguar debido a un problema de conservación diferencial. Aunque su vida útil es muy reducida y únicamente servirían para cobrar piezas de caza menor, la rapidez con que se fabrican los hacen bastante rentables, (Olsen,
1973). Es relativamente fácil encontrar fragmentos rectos de forma natural y el centro hueco de la caña es ideal para insertar una punta de flecha larga y estrecha (Elmer, 1952).

Las puntas de flecha presentan un gran poliformismo, aunque generalmente la morfología y los materiales de las mismas están íntimamente unidos a su funcionalidad. Así, hay puntas de madera muy aguzadas, de metal y de piedra; puntas redondeadas, transversales, etc.

"Desde el punto de vista aerodinámico, la forma idónea es el cilindro rematado en cono (forma de huso o bala). Pero esta forma (que es la utilizada en competición) no es útil para la caza, ya que al mismo tiempo que penetra en los tejidos del animal hace -el astil- de tapón evitando el desencadenamiento de la hemorragia. Entonces, la forma idónea para la caza es aquella que teniendo un desarrollo aerodinámico suficiente permite crear una herida amplia que no sea taponada por el astil. La forma ideal es aquella radial con aspas de sección hemifusiforme que dejará en el animal una zona cruenta amplia e imposible de taponar por el astil. Dentro de este grupo, la única accesible a su fabricación con los medios disponibles en el Paleolítico es la plana (dos aspas)" (Rasilla, 1989).

Las puntas cónicas y largas de un diámetro similar al del astil penetran muy mal en los tejidos animales, ya que no generan un corte limpio en la herida y el rozamiento con los bordes de la misma provocan que se frene. Así, las puntas de sílex y, sobre todo, las de obsidiana penetran mejor en los tejidos que las de acero. Además, el ángulo que forma el extremo distal de la punta es un elemento importante que influye en la penetración del proyectil: cuando mayor es el ángulo, mayor es la posibilidad de que la punta rebote en el blanco. Estudios experimentales demuestran que las piezas con ángulos superiores a $56^{\circ}$ tienen una gran probabilidad de rebotar en el blanco (Odell y Cowan, 1986).

El último proceso en la fabricación de la flecha es el emplumado de la misma. Este consiste en varias plumas colocadas en el extremo proximal del astil para ayudar a estabilizar el proyectil durante el vuelo y a trazar una trayectoria rectilínea. La longitud y anchura de las plumas deben ser proporcionales a la longitud y peso de la flecha para evitar un rozamiento innecesario que provoque una merma en la capacidad de alcance y de penetración. Existen dos formas de emplumado: el radial, en donde 3 ó 4 plumas partidas por la mitad, conservando el

T. P., 56, n. ${ }^{\circ} 1,1999$ 
raquis, se unen por separado al astil quedando equidistantes entre sí y, la tangencial, en donde dos plumas enteras se colocan enfrentadas sobre el astil. Lógicamente, en el material arqueológico de la secuencia europea no existen evidencias directas del uso de flechas emplumadas, aunque los primeros indicios de dardos con un posible emplumado para ser lanzados con propulsor proceden del Magdaleniense (Mayet y Pissot, 1915). Teniendo en cuenta la complejidad de las operaciones llevadas a cabo en la fabricación de los astiles mesolíticos, es probable que éstos tuvieran plumas. No obstante, este elemento no es imprescindible para que la flecha pueda ser disparada desde el arco.

El emplumado del astil y la sujeción de los proyectiles al mismo, aunque estos últimos pueden estar únicamente atados con fibras vegetales o tendones animales, presupone el uso de elementos adhesivos. Las colas puramente orgánicas han desaparecido, aunque las estrías y acanaladuras del utillaje óseo atestiguan su empleo al menos desde el final del Solutrense. Las diferentes investigaciones sobre este tema han demostrado que las resinas vegetales son bastante eficientes para la sujeción del utillaje. La resina de abedul (Betula alba) se ha mostrado mucho más eficaz y resistente que la proporcionada por las coníferas (Olsen, 1973). Sin embargo, la combinación de resina de pino (3 partes) y cera (1 parte), añadiendo polvo de ocre como emulsionante, forma una mezcla homogénea parecida al lacre. Para ligar estos tres elementos se necesita una fuente de calor no muy elevada, alrededor de $120^{\circ}$, aunque si la resina de pino se sustituye por la de abedul el punto de fusión es más bajo, (Allain y Rigaud, 1989). La efectividad de este pegamento queda demostrada por su empleo hasta principios de siglo para fijar útiles metálicos en una espiga de madera. La presencia de resinas vegetales se documenta por primera vez en astiles y proyectiles del neolítico lacustre del norte y centro de Europa (Déchelette, 1908; Mathiassen, 1948; Troels-Smith, 1959; Vogt, 1952; Clark, 1963; Rozoy, 1978). No obstante, en los yacimientos magdalenienses de Garenne (Allain y Descout, 1957), Lascaux (Leroi-Gourhan y Allain, 1977) y Pincevent (Leroi-Gourhan, 1983) aparecen elementos líticos y óseos con restos de ocre como posible testimonio indirecto del uso de este tipo de adhesivo. Tampoco se puede descartar el empleo de colas realizadas a partir de piel, hueso o espinas de pescado mediante una cocción más o menos prolongada, añadiendo como aglutinante un poco de cal viva, aunque lamentablemente no dejan ninguna huella identificable de su posible uso. Entre todas ellas, la más efectiva es la llamada "Cola de Moscovia" obtenida a partir de la vejiga natatoria del esturión (Allain y Rigaud, 1989).

\section{CONDICIONAMIENTOS BALÍSTICOS PARA EL EMPLEO DE PUNTAS DE FLECHA EN ACTIVIDADES CINEGÉTICAS}

Tradicionalmente, el peso tanto de los astiles como de las puntas se ha considerado como el indicador más válido para ponderar la funcionalidad del proyectil como punta de flecha o punta de dardo (Fenega, 1953). En muchas ocasiones el término "punta de proyectil" se ha usado como un eufemismo ante la imposibilidad de establecer una interpretación funcional precisa. Para obtener la máxima estabilidad direccional y una penetración efectiva el peso de la punta debe repartirse a lo largo del astil, ya que sino el vuelo de la flecha es muy corto y de escasa potencia. Otros autores, sin embargo, concluyen que el área de enmangue es el indicador funcional más importante ya que esta zona debe correlacionarse con el diámetro del astil (Forbis, 1960; Wyckoff, 1964). Otro sistema de análisis ha sido la experimentación directa con arcos y propulsores, como los trabajos de J. Browne (1938 y 1940) quien demostró que las puntas de flecha de unos $5 \mathrm{~cm}$. de longitud son las más efectivas. Las flechas conocidas por los pueblos primitivos actuales pesan, por regla general, entre 20 y 30 gr. aproximadamente (Pope, 1962). Sin embargo, este mismo autor ha usado flechas de hasta 42 gr. de peso, de los que 14 gr. corresponden a la punta de proyectil, con arcos simples y se han mostrado de gran eficacia para abatir presas de gran tamaño, como cérvidos.Aunque J.G. Rozoy (1978) considera que las puntas de flecha para los arcos mesolíticos no deberían sobrepasar los 5 gr. y el peso total de las flechas apenas superaría los 30 gr., hay ejemplos etnográficos de primitivos actuales africanos, como la tribu Tindiga (Kohl-Larsen, 1958), que utilizan flechas de 65 gr. a 100 gr. con puntas de metal.

Asimismo, los trabajos con arcos experimentales llevados a cabo por J. Browne (1938 y 1940) demuestran que es factible lanzar puntas de proyectil, supuestamente de jabalina, de hasta $60 \mathrm{~mm}$. de longitud y hasta 10 gr. de peso enmangadas en astiles de flecha con un peso total de unos 40 gr. No obstante, este investigador también señala que los 
proyectiles que sobrepasan los $35 \mathrm{~mm}$. de longitud tienen una mayor posibilidad de quebrarse y, por lo tanto, son menos efectivos.

En el estudio con materiales etnográficos y arqueológicos realizado por D. Hurst (1978) sobre 118 puntas de flecha y 10 puntas de jabalina pertenecientes a 12 pueblos de diferentes tribus deAmérica del Norte, el peso máximo que alcanzan las primeras es de 17,4 gr., aunque solo 5 ejemplares sobrepasan los 5 gr. Asimismo, en este trabajo se constata una fuerte correlación entre el peso de la punta y el tamaño de la flecha: los astiles más grandes tienden a albergar las puntas más pesadas. El análisis multivariante que realiza entre los dos tipos de proyectiles da como resultado que la anchura del elemento de enmangue es el valor discriminante más importante entre las puntas de flecha y las puntas de dardo. En estas últimas, la anchura del punto de enmangue siempre sobrepasa los $11 \mathrm{~mm}$., mientras que en las puntas de flecha casi nunca rebasa esta medida. En los lanzamientos con distintos arcos experimentales y un propulsor llevados a cabo por C. Bergman, E. McEven y R. Miller (1988), utilizaron para este último una jabalina de $152 \mathrm{~cm}$. de longitud con un proyectil bifacial y un peso total de 195 gr. En el arco simple sioux de doble curvatura y de $112 \mathrm{~cm}$. de largo, realizado en hickory (Carya glabra), el peso de la flecha era de 30 gr. Para el arco simple de tipo africano, realizado en una sola pieza de madera dura no identificada, de $173 \mathrm{~cm}$. de largo, la flecha alcanzaba los 40 gr. Los ejemplares más pesados, 90 gr., fueron utilizados tanto en la réplica de longbow medieval simple de $293 \mathrm{~cm}$. de longitud, fabricado mediante dos brazos de tejo empalmados en el centro, y en un arco compuesto angular egipcio de cuerno, madera y tendón. Todas las flechas utilizadas fueron réplicas de piezas originales. Estos datos demuestran que a medida que el arco es tecnológicamente más desarrollado, permite el uso de flechas de mayor peso ya que la energía cinética almacenada es mucho mayor.

El material arqueológico europeo revela también la existencia de proyectiles de muy escaso peso. Por ejemplo, las puntas tardenoienses (trapecios) alcanzan los 2 gr. (Rozoy, 1978). Las puntas de Istres no superan los 1,5 gr. (comunicación personal de Escalon de Fonton a Rozoy, 1978). Las puntas azilienses de Roc d'Arbeilles tienen un peso medio de 1,76 gr., mientras que las puntas de Malaune y de Laugerie Basse del mismo yacimiento son aún más ligeras: en torno a 0,7 gr. (comunicación personal de Champagne a Rozoy, 1978). Por último, casi todas las puntas bifaciales neolíticas se sitúan en torno a los 2 gr. de peso (Baye, 1874a,b; Harmand, 1952).

Los factores más importantes que afectan tanto a la velocidad que alcanza la flecha como la del disparo son el diseño del proyectil y del arco, así como el peso del primero y las materias primas empleadas en la fabricación del segundo. A lo largo del vuelo de la flecha hay una pérdida progresiva de la energía cinética inicial que se traduce en una disminución de la velocidad de partida en función de las fuerzas de rozamiento y gravedad que están íntimamente relacionadas con el peso y la forma del astil y la punta. Por lo tanto, la eficacia del lanzamiento depende de la cantidad de energía cinética de la flecha en el momento del impacto y en que parte del animal se localiza éste. En la penetración de la piel y de la aponeurosis superficial se pierde una parte importante de esta energía, mientras que el impacto o rozamiento del proyectil contra huesos, tendones o cartílagos puede frenar considerablemente la longitud de penetración o parar totalmente la misma. La velocidad inicial del lanzamiento depende de tres variables básicas: la magnitud de la fuerza desarrollada por el arco, la velocidad de transmisión de la misma a la flecha y la masa a desplazar. $\mathrm{La}$ energía potencial que acumula el arco y que luego se transfiere a la flecha es menor en los prototipos de madera simples de una sola pieza que en los arcos reforzados o compuestos. El excesivo peso de la punta o su defectuosa distribución a lo largo del astil provoca una disminución de la velocidad y por lo tanto una pérdida de eficacia. Asimismo, la velocidad de expansión de los brazos y de la cuerda determinará en gran medida la cantidad de energía cinética inicial que se transfiere a la flecha.

Los primeros trabajos experimentales sobre el alcance de los arcos, es decir, la distancia a la que se dispara la flecha, se deben a S. Pope (1923 y 1974). Aunque, como todo estudio pionero, presenta algunas connotaciones que invalidan en parte los resultados obtenidos. Por un lado, no se tuvo en cuenta la función para la que fueron concebidos los diferentes tipos de arcos y, por otro, las réplicas experimentales no siempre se fabricaron con los materiales usados en el original. Además, las flechas utilizadas no se correspondían en todos los casos con el registro arqueológico o etnográfico. El artículo, ya clásico, de C. Bergman, E. McEven y R. Miller (1988) sobre la comparación de la velocidad de diferentes proyectiles y arcos es el que cuenta con una documentación más rigurosa y precisa.Además de una fiel réplica de los distintos tipos de arcos y fle-

T. P., 56, n. ${ }^{\circ} 1,1999$ 
chas, se usó un sistema informatizado de medición de la velocidad inicial de los proyectiles de una gran precisión, con un equipo especialmente diseñado para ello de la Royal Ordenance Small Arms Division. Las experimentaciones con los arcos más primitivos, que se pueden asimilar a los ejemplares mesolíticos y neolíticos, fueron las realizadas con un arco sioux simple de hickory y un arco africano, también simple, de una madera dura desconocida. El tipo más lento fue el primero, ya que con una tensión de $25 \mathrm{~kg}$. logro imprimir a una flecha de $30 \mathrm{gr}$. una velocidad inicial de $30 \mathrm{~m} / \mathrm{s}$. En este caso, la materia prima tuvo una gran influencia ya que es una madera débil y rebota rápidamente cuando se libera la tensión de la cuerda. En el segundo tipo, con una tensión de $24 \mathrm{~kg}$. una flecha de $40 \mathrm{gr}$. alcanzó una velocidad inicial de $35 \mathrm{~m} / \mathrm{s}$. Los lanzamientos experimentales demostraron que el peso y diseño de la flecha inciden directamente en la velocidad inicial. Las flechas más ligeras alcanzan una mayor velocidad inicial. Sin embargo, sólo los ejemplares más pesados son capaces de absorber toda la energía potencial que genera el arco (Klopsteg, 1947). Los primeros arcos aparecidos en el registro arqueológico europeo debieron tener una mayor tensión que los ejemplares analizados; ya que sus largas palas agotan una parte importante de la energía potencial almacenada al tensarse.

No obstante, la longitud que alcanza una flecha lanzada mediante un arco simple puede ser muy elevada. Por ejemplo, J. Browne (1938 y 1940) constata que con una flecha de unos 40 gr. se puede abatir un alce adulto a una distancia de $175 \mathrm{~m}$., aunque se emplearon puntas metálicas. En los lanzamientos de S. Pope (1962 y 1974) con longbow medievales de tejo se alcanzaron distancias de hasta $400 \mathrm{~m}$. No obstante, con réplicas de arcos simples solo se llegó a los $200 \mathrm{~m}$. En las actividades cinegéticas este factor no es del todo determinante, ya que una distancia de entre $40 \mathrm{~m}$. y $50 \mathrm{~m}$. es suficiente para no alertar a la presa y tener una precisión bastante buena. El impacto que registra a esta distancia el proyectil a unos $30 \mathrm{~m} . / \mathrm{s}$. es de $3,5 \mathrm{~kg}$. aproximadamente (Rozoy, 1978).

\section{LAS PUNTAS LIGERAS DE PROYECTIL DEL SOLUTRENSE EXTRACANTÁBRICO}

Una vez establecidas las principales características técnicas, morfométricas y balísticas que inci- den en el uso del arco y la flecha y, que condicionan el nivel tecnológico que se debe alcanzar para un hipotético inicio de la arquería prehistórica, el siguiente paso será analizar los testimonios que pudieran evidenciar el uso del arco como sistema de propulsión de las puntas ligeras de proyectil del Solutrense Extracantábrico.

En primer lugar, nos encontramos ante un hecho incontestable, la falta de evidencias materiales en todo el continente europeo antes del Mesolítico. Sin embargo, la conservación diferencial de los elementos que conforman el registro arqueológico y las circunstancias excepcionales que han permitido la preservación de los primeros ejemplares, no presupone la aparición del arco en este momento. Por un lado, la perfección formal tanto de las palas como de la empuñadura de los arcos mesolíticos hace pensar que no es posible su súbita aparición en el acervo cultural y tecnológico de uno o varios grupos, sino que más bien, es el resultado de una evolución gestada en momentos anteriores y producto de un largo proceso de experimentación. Por otro lado, la complejidad del sistema de fabricación y el control de todos los factores que inciden en una mayor o menor rentabilidad cinegética del arco, también hacen pensar en un proceso evolutivo largo y costoso en donde hay una gradación temporal en los avances técnicos conseguidos. Igual que no es factible pensar en la invención del arco compuesto sin la experiencia previa del arco reforzado, del mismo modo, la aparición del arco simple no hubiera sido posible sin prototipos más rudimentarios, en donde el concepto de la transmisión de la energía no se materializa de forma totalmente correcta. Asimismo, la elaboración de astiles del corazón de árboles viejos, sin nudos ni deformaciones, y divididos en dos partes intercambiables para reponer únicamente la pieza distal cuando ésta se fracturara por el impacto o durante la penetración, lo que supone una gran inversión de tiempo y trabajo, además de un esquema mental complejo, presuponen un proceso de experimentación previa que da como resultado una progresiva optimización de los patrones de trabajo y de la eficacia del instrumental cinegético.

Todas estas consideraciones, nos llevan a plantear la hipótesis de la aparición del arco durante el Solutrense Superior, por lo menos, en la vertiente mediterránea de la Península Ibérica, cuando surgen los primeros proyectiles susceptibles de ser puntas de flecha. Lógicamente los primeros prototipos de "arcos" estarían realizados, o bien de ramas más o 
menos rectas y gruesas, o bien de árboles jóvenes, ambos con una modificación muy somera del soporte original. Del mismo modo, los astiles serían fabricados de una sola pieza, a partir de ramas rectas y delgadas de árboles también jóvenes, y posiblemente no estarían emplumados. El análisis polínico con que cuenta la Cueva de Ambrosio (Ripoll, 1988) demuestra la existencia en el entorno de este yacimiento de las materias primas necesarias para la fabricación de arcos y astiles.Así, los primeros pudieron estar realizados en madera de olmo (Ulmus), nogal (Juglans) o tilo (Tilia) y los segundos a partir de ramas de pino (Pinus), avellano (Corylus) o incluso de boj (Buxus).

Lógicamente, todas estas afirmaciones quedarían en meras especulaciones si las únicas evidencias posibles del uso del arco en el Solutrense, las puntas de aletas y pedúnculo y las puntas de muesca de retoque abrupto de tipo mediterráneo, no se adaptaran a las exigencias balísticas para ser puntas de flecha. Como hemos visto, los proyectiles que sobrepasan los $35 \mathrm{~mm}$. de longitud son menos efectivos al tener una mayor posibilidad de quebrarse. Asimismo, las puntas de flecha no sobrepasan los 5 gr. de peso y la anchura de la zona de enmangue no supera los $11 \mathrm{~mm}$. de longitud. Por otro lado, el diámetro de los astiles normalmente se sitúa entre los $8 \mathrm{~mm}$. y los $9 \mathrm{~mm}$.

En las puntas de aletas y pedúnculo, la media aritmética de la longitud total es de $37,11 \mathrm{~mm}$. en la Cova del Parpalló y de 41,09 mm. en la Cueva de Ambrosio, con una amplitud de valores que oscila entre los $67 \mathrm{~mm}$. y los $14 \mathrm{~mm}$. (Fig. 2A). Sin embargo, la media aritmética de la longitud de la punta (una vez embutido el pedúnculo en el astil) es de $28,58 \mathrm{~mm}$. en el yacimiento valenciano y de 32,29 $\mathrm{mm}$. en el almeriense, con unos valores extremos que se sitúan entre $61 \mathrm{~mm}$. y $11 \mathrm{~mm}$. (Fig. 2B). En las puntas de muesca, la media aritmética de la longitud total oscila entre los $35,74 \mathrm{~mm}$. de la Cueva de Ambrosio y los 32,95 mm. de la Cova del Parpalló. La amplitud de los guarismos máximos y mínimos es de $69 \mathrm{~mm}$. y $15 \mathrm{~mm}$. respectivamente (Fig. 3A). La media aritmética de la longitud de la punta es de $28,74 \mathrm{~mm}$. en la estación almeriense y de 24,4 $\mathrm{mm}$. en la valenciana, llegando a alcanzar hasta los $59 \mathrm{~mm}$. y en el otro extremo los $9 \mathrm{~mm}$. (Fig. 3B). Así pues, la longitud de la punta de ambos tipos de proyectiles se sitúa en la mayoría de las ocasiones por debajo de los $35 \mathrm{~mm}$., medida a partir de la cual se reduce la efectividad de las puntas al aumentar el riesgo de fractura.

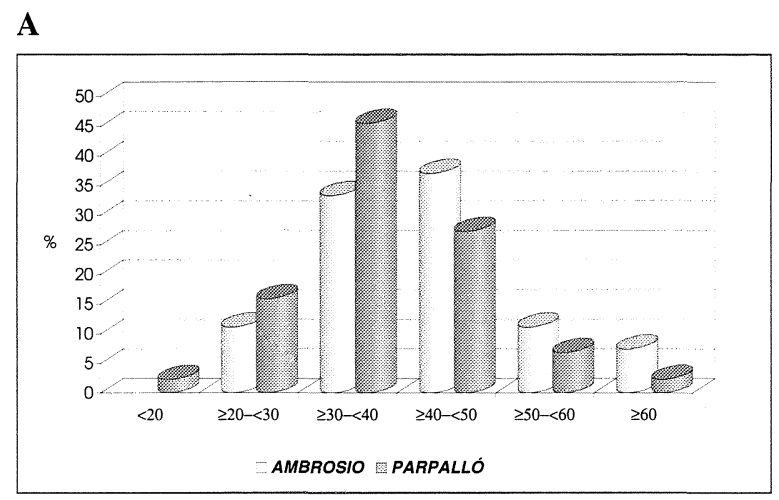

B

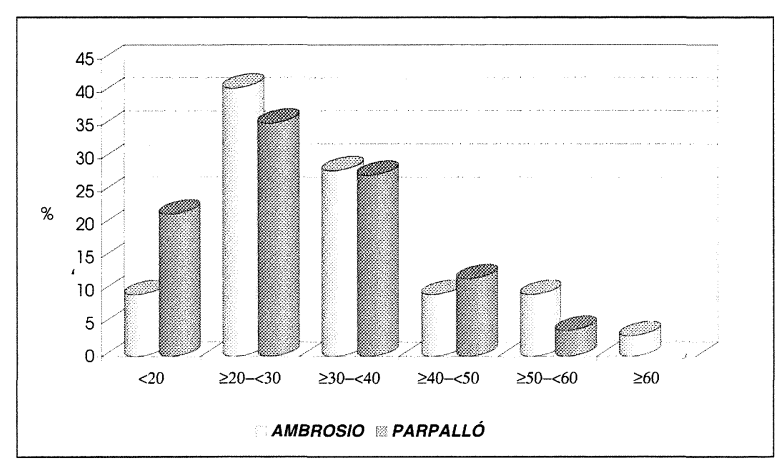

Fig. 2. Punta de aletas y pedúnculo. A: Longitud total. B: Longitud de la punta.

En cuanto a la zona de enmangue, en las puntas de aletas y pedúnculo la media aritmética de la anchura de este último elemento, el pedúnculo, es de $5,65 \mathrm{~mm}$. en la Cova del Parpalló y de 6,82 mm. en la Cueva deAmbrosio. En el total de ambos conjuntos los valores extremos oscilan entre los $12 \mathrm{~mm}$. y los $2 \mathrm{~mm}$. No obstante, entre los $5 \mathrm{~mm}$. y los $9 \mathrm{~mm}$. se agrupa el $81,84 \%$ y el $60,61 \%$, respectivamente, de los ejemplares de cada yacimiento (Fig. 4A). En cuanto a las puntas de muesca, la media aritmética de la anchura del elemento de enmangue es de $8,04 \mathrm{~mm}$. en la Cova del Parpalló y de 7,62 mm. en la Cueva de Ambrosio. La amplitud de valores se sitúa entre los $16 \mathrm{~mm}$. y los $2 \mathrm{~mm}$. Sin embargo, el $97,36 \%$ de los proyectiles del yacimiento valenciano se coloca entre los $5 \mathrm{~mm}$. y los $10 \mathrm{~mm}$., mientras que en las estación almeriense esta proporción es del $87,96 \%$ (Fig. 4B). Estos valores, para ambos tipos de proyectiles, en la inmensa mayoría de la población están dentro de los parámetros establecidos para las puntas de flecha.

En cuanto a su peso, los datos proceden de la suma de las muestras de ambos yacimientos, que han sido tratados de forma conjunta. En el caso de 


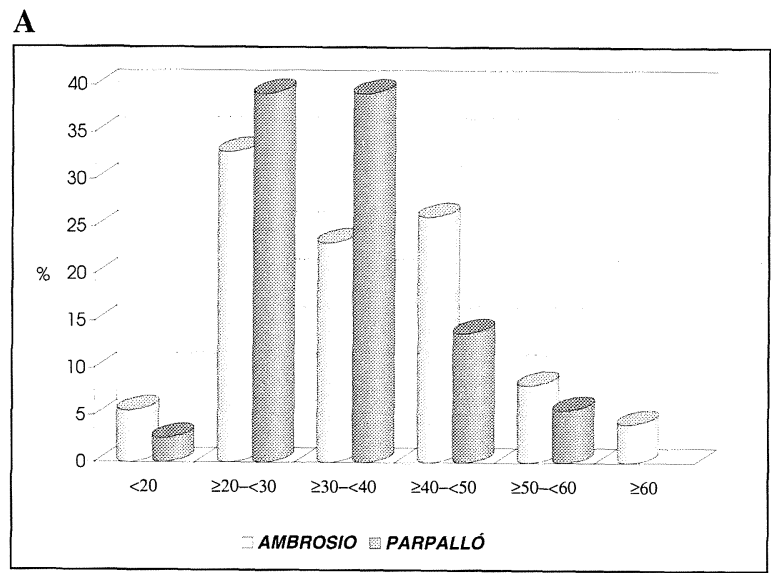

B

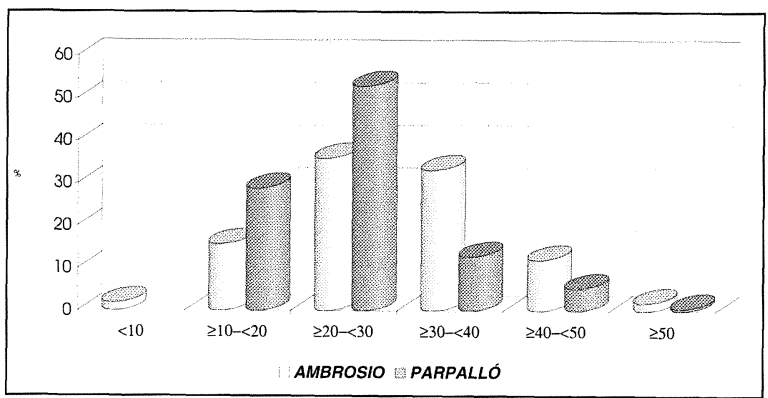

Fig. 3. Punta de muesca. A: Longitud total. B: Longitud de la punta.

A

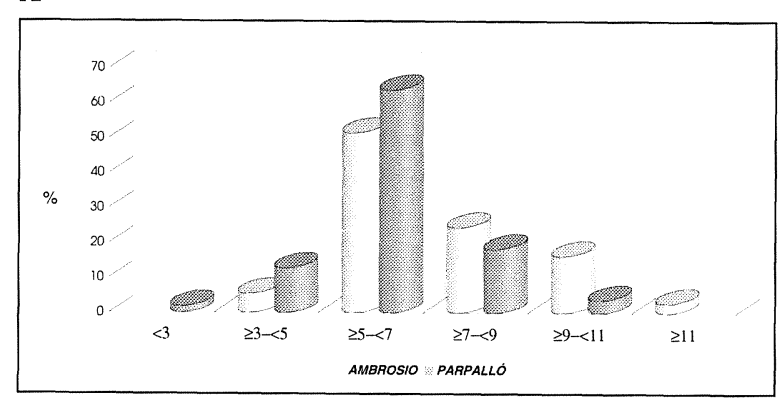

B

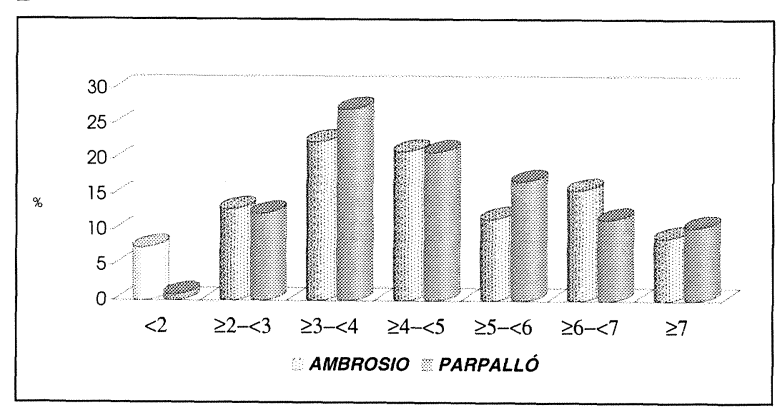

Fig. 4. Anchura del elemento de enmangue. A: Anchura del pedúnculo. B: Anchura de la muesca. las puntas de aletas y pedúnculo, únicamente 17 piezas no presentaban fracturas. La media aritmética de esta magnitud es de 1,33 gr., siendo el proyectil más ligero de tan solo 0,31 gr. y el más pesado de 3,5 gr. Si a estos ejemplares se añaden las puntas en donde los procesos de fragmentación no tienen gran trascendencia, afectan a menos $\mathrm{de}^{1 /} / 3$ de la longitud total de la pieza, el número asciende a 68 . En este conjunto, la media aritmética es algo superior, 1,66 gr., mientras que el valor máximo se sitúa en 6,9 gr. En las puntas de muesca el número de proyectiles sin fracturas es de 110. La media aritmética del peso de este grupo es de 0,76 gr., con valores máximos y mínimos que oscilan entre los 5,9 gr. y los 0,1 gr. Si se consideran además las puntas con fracturas de escasa entidad (340 piezas), como en el caso anterior, las fracturas afectan a menos $\mathrm{de}^{1} / 3 \mathrm{de}$ su longitud total, la media aritmética es incluso algo menor (0,74 gr.). La amplitud de los valores extremos es la misma. Estos datos atestiguan que en la inmensa mayoría de las ocasiones el peso de ambos tipos de proyectiles no superan los $5 \mathrm{gr}$.

Así, las características morfométricas del registro arqueológico se adaptan en la práctica totalidad de la muestra de estudio a las exigencias intrínsecas que los proyectiles necesitan para realizar su función de punta de flecha. Por último, las morfologías características de ambos morfotipos determinan en gran medida los sistemas de sujeción al astil. Como se desprende del estudio de los procesos de fragmentación de las puntas ligeras de proyectil, éstas debieron ser pegadas y atadas a los astiles, (Muñoz, 1997, e.p.). El análisis polínico del yacimiento de la Cueva de Ambrosio (Ripoll, 1988) constata la presencia de especies arbóreas cuyas resinas son susceptibles de ser utilizadas como elementos adhesivos. Este sería el caso del pino ( $P i$ $n u s$ ) y del abedul (Betula). Esta última es considerada como la más idónea para la sujeción de elementos líticos a los astiles de madera. Por otro lado, la presencia de numerosos vestigios de ocre,así como el descubrimiento, en la capa 2-2bis de la Microestratigrafía, de una plaqueta con restos de este material asociado a estructuras de combustión (Ripoll et alii, 1997), podría indicar el uso de resina, ocre y cera para la elaboración de pegamentos de gran consistencia. Además, los proyectiles pudieron ser atados a los astiles mediante fibras vegetales y/o tendones animales.

En el caso de las puntas de aletas y pedúnculo, el sistema de enmangue que proponemos se realizaría mediante un cajeado en el extremo distal del 
astil, perpendicular al eje longitudinal del mismo (Fig. 5). En esta muesca se introduciría el pedúnculo hasta el punto de unión con el arranque de las aletas, sujetándolo al astil con resinas y/u otros elementos adhesivos. El retoque plano del pedúnculo crea una superficie algo rugosa que facilita una mayor y mejor adherencia del pegamento entre el elemento de enmangue y la superficie fibrosa del astil. Por último, se realizaría un amarre circular a lo largo de toda la zona del astil donde queda embutido el pedúnculo para reforzar el enmangue. Por lo tanto, la anchura del pedúnculo marcaría el diámetro mínimo que puede alcanzar el astil, aunque no debe descartarse una morfología cónica del mismo para adaptarse mejor a las dimensiones del pedúnculo o, simplemente, astiles más anchos.

Las aletas actuarían como elemento de tope entre la punta y el astil, impidiendo que la primera retroceda en el momento del impacto, lo que explicaría el alto porcentaje de fracturas a la altura del pedúnculo y en la parte inmediatamente superior (Fig. 5). También, estos elementos disminuyen el rozamiento durante la penetración, ya que sobresalen más que la zona atada, en la mitad del diámetro del astil. Las aletas en ángulo recto solo tendrían esta función. Sin embargo, las morfologías triangulares y en gancho, además, impedirían que el proyectil pudiera salirse del blanco una vez dentro, penetrando aún más en los tejidos en el caso que el animal intentara deshacerse de la flecha frotándose contra algún elemento del entorno. Por lo tanto, las posibilidades de recuperar el proyectil son $\mathrm{mu}$ cho más altas.

El retoque plano de la punta genera un filo extremadamente cortante $y$, por tanto, con una gran capacidad de desgarrar tejidos, es decir, es capaz de provocar importantes hemorragias y heridas internas en el animal que le pueden causar la muerte en un corto espacio de tiempo o dejarlo muy debilitado. Además, el ángulo del extremo distal del proyectil asegura un gran coeficiente de penetración. Así, la media aritmética de esta magnitud es de $18,72^{\circ}$ en la Cova del Parpalló y de $15,52^{\circ}$ en la Cueva de Ambrosio. Los ángulos máximos nunca sobrepasan $\operatorname{los} 30^{\circ}$ y los valores mínimos se sitúan en $5^{\circ}$. Los ángulos más abiertos quedan bastante alejados de los $56^{\circ}$, cifra a partir de la cual las posibilidades que tiene la punta de rebotar en la presa y no penetrar en ella son muy elevadas (Fig. 7A).

La morfometría de las puntas de aletas y pedúnculo, plana y con dos aspas, es la más idónea -teniendo en cuenta el grado de desarrollo tecnoló-

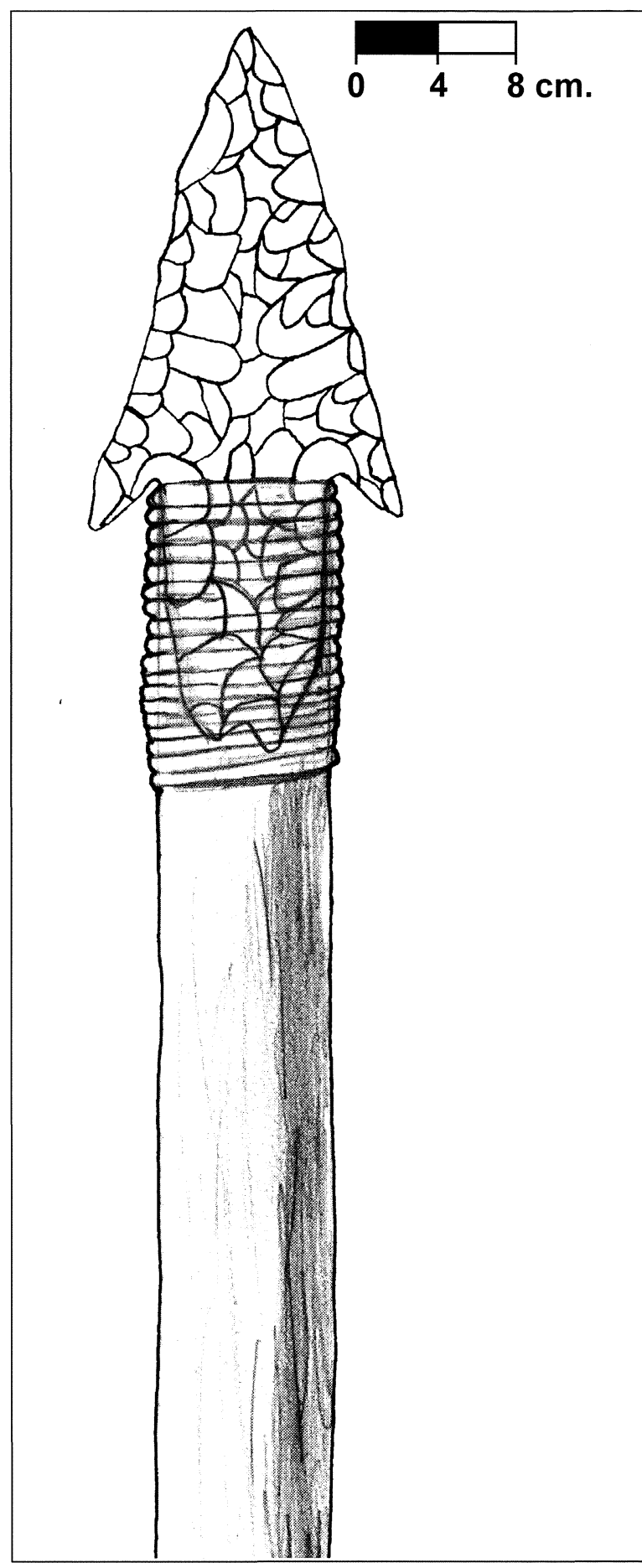

Fig. 5. Sistema de enmangue propuesto para la punta de aletas y pedúnculo.

gico alcanzado en el Solutrense-para la función de punta de flecha. Resulta. entonces, difícil de entender porque a lo largo del Solutrense Superior Evo- 
lucionado hay un progresivo abandono, hasta casi su total desaparición, de este morfotipo en favor de la punta de muesca de tipo mediterráneo. Si la forma ideal de la punta de flecha, para las actividades cinegéticas, es la radial con cuatro aspas y de sección hemifusiforme, y la punta de aletas y pedúnculo solo presenta dos, parece lógico pensar que la supresión de las mismas del registro arqueológico está relacionada con la aparición de una morfología más efectiva ( 3 ó 4 aspas) o cuyo proceso de fabricación sea más sencillo.

La hipótesis de enmangue que hemos elaborado para la punta de muesca cumple estas dos premisas (Fig. 6). Este tipo de proyectiles estaría enmangado en un astil circular cuyo extremo distal sería puntiagudo, con una progresiva disminución del diámetro del mismo (Fig. 6A). Las puntas de muesca irían embutidas en el extremo distal del astil y paralelas al eje longitudinal del mismo. Mediante un cajeado en la madera, cuya profundidad disminuiría a medida que se llega al extremo distal, la muesca quedaría totalmente introducida en el astil. Este sistema de enmangue permite crear una punta de 2, 3 ó 4 aspas equidistantes entre sí (Fig. 6B).

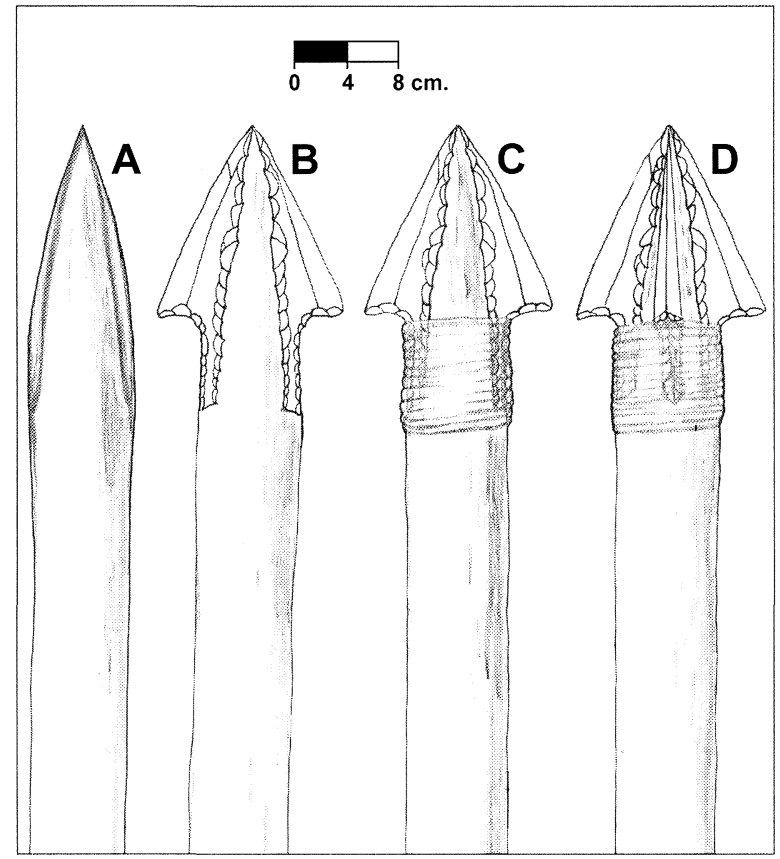

Fig. 6. Sistema de enmangue propuesto para la punta de muesca. A: Cajeado del extremo distal del astil. B: Sujeción de los proyectiles en el astil mediante elementos adhesivos. C: Sujeción de los proyectiles en el astil mediante un amarre circular. D: Implementación de tres o cuatro elementos.

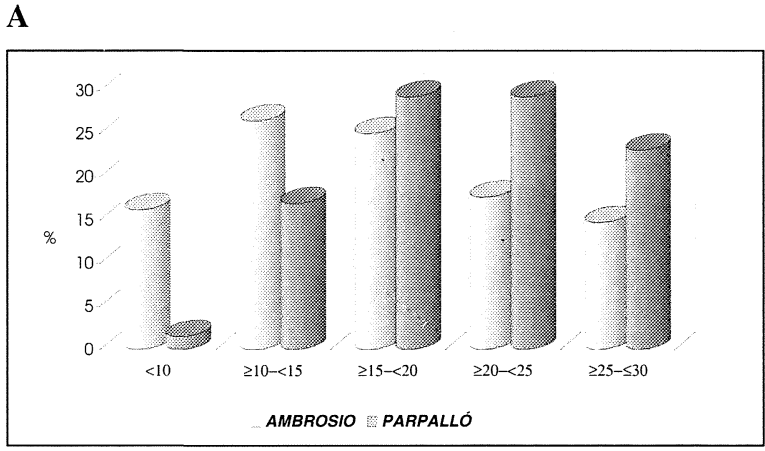

B

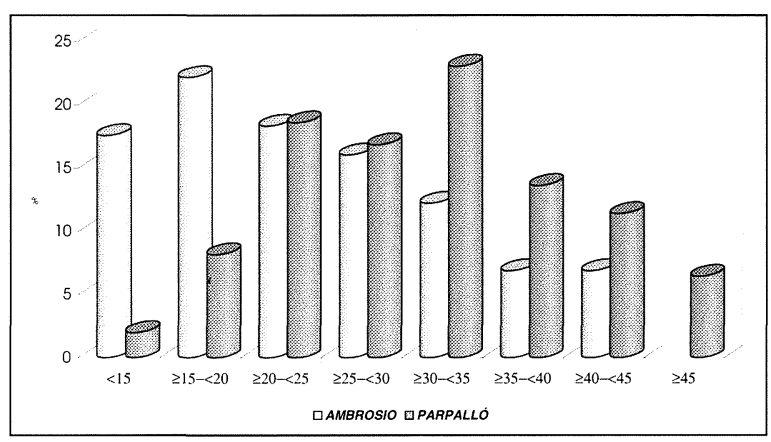

Fig. 7. Ángulo del extremo distal del proyectil. A: Punta de aletas y pedúnculo. B: Punta de muesca.

El borde opuesto a la muesca generalmente presenta un retoque abrupto con varias series de levantamientos que crea una superficie rugosa. Como en el caso anterior, esto otorga una mayor efectividad a los elementos adhesivos, creando una sujeción bastante fuerte. El borde contrario presenta un filo natural, o parcialmente retocado, que también asegura una gran capacidad de corte. Asimismo, la concavidad de la muesca permite atar los distintos proyectiles, a lo largo de toda la muesca, mediante un amarre circular sin que se produzca ningún rozamiento suplementario durante la penetración (Fig. 6C).

Los astiles con dos puntas de muesca enfrentadas y equidistante entre sí tendrían las mismas propiedades balísticas y cinegéticas que los enmangados con puntas de aletas y pedúnculo. Sin embargo, la fabricación de una punta de muesca es mucho más sencilla, requiere una menor inversión de tiempo y tiene una mayor posibilidad de éxito en su ejecución, con un mayor control de las variables morfométricas para su función como punta de flecha, que la punta de aletas y pedúnculo. Los astiles con 3 ó 4 puntas aumentan la efectividad del disparo, adquiriendo morfologías similares a las puntas de 
flecha realizadas en metal y utilizadas hasta la aparición de las primeras armas de fuego (Fig. 6D).

La implementación de hasta 4 puntas de muesca en un mismo astil no varía las características analizadas y que hacían teóricamente factible su uso como punta de flecha. Tanto la longitud total como la longitud de la punta y la anchura del punto de enmangue no modifican sus propiedades balísticas. Únicamente, el peso conjunto de varias puntas podría descalificar esta hipótesis. Sin embargo, como la media aritmética de esta magnitud es menor de 1 gr., la mayor parte de las flechas podrían contar con 3 y 4 puntas sin que el peso total sobrepasara los 5 gr., es decir, la barrera teórica a partir de la cual el peso de la punta es excesivo para repartirse de forma homogénea a lo largo del astil. Por otro lado, el ángulo del extremo distal de la punta tampoco presenta modificaciones importantes, ya que su medición supone la mitad del ángulo obtenido para las puntas de aletas y pedúnculo. Así, la media aritmética del ángulo de las puntas de muesca de la Cova del Parpalló es de $28,56^{\circ}$ mientras que en la Cueva de Ambrosio es de $21,96^{\circ}$. Los valores máximos de esta magnitud alcanzan los $50^{\circ}$ y los mínimos los $10^{\circ}$. En la totalidad de los casos el ángulo del extremo distal nunca supera los $56^{\circ}$ (Fig. 7B).

\section{CONCLUSIONES}

La dinámica evolutiva que siguen las puntas ligeras de proyectil del Solutrense Extracantábrico parece tener una continuación lógica en las etapas culturales posteriores. Así, la punta de aletas y pedúnculo es sustituida por la punta de muesca y no volverá a reaparecer hasta el Neolítico, cuando el grado de desarrollo tecnológico permita la existencia de sistemas de lanzamiento que acumulen una mayor energía cinética inicial, es decir, arcos de mayor potencia. Las puntas de muesca, que perviven en el Magdaleniense, el Epipaleolítico y el Neolítico aunque su importancia sea cada vez menor, serían sustituidas por las hojitas de dorso. La presencia cada vez más numerosa de este tipo de utillaje junto con el ascenso de las puntas de proyectil en asta y hueso, muchas de ellas con una acanaladura central, se documenta a partir de los momentos finales del Solutrense. Así, no parece descabellado pensar en la existencia de proyectiles compuestos en donde las hojitas de dorso irían pegadas en las azagayas, generando puntas de 4 aspas, o incluso embutidos en los mismos astiles a modo de arpones dentados. Además, estos elementos líticos de proyectil presentan, aún si cabe, una mayor facilidad y rapidez en su fabricación. Por otro lado, el enmangue de la azagaya preservaría al extremo distal del astil de fracturas en esta zona, su parte más débil, siendo más fácil fabricar una nueva azagaya que un astil. Por último, las hojitas de dorso serían sustituidas por los microlitos geométricos del Epipaleolítico.

Sin embargo, la aparición del arco y la flecha no significa necesariamente la exclusión de otros sistemas de lanzamiento ya existentes para el desarrollo de las actividades cinegéticas concretas. Así, a pesar del conocimiento de la tecnología del arco y la flecha, los aztecas usaron propulsores para pescar y cazar aves acuáticas (Nuttall, 1891). Los esquimales también usaron el propulsor para cazar aves y focas desde pequeñas embarcaciones (Stirling, 1960). En este sentido, el propulsor, documentado desde el final del Solutrense en materias duras animales (Cattelain, 1988, 1989), pudo haber tenido precedentes realizados en materiales orgánicos como la madera y que habrían desaparecido por un problema de conservación diferencial. Este sistema de propulsión podría haber sido perfectamente utilizado para lanzar dardos que tendrían como puntas de proyectil hojas de laurel.

La principal ventaja del arco frente al propulsor radica en la mayor rapidez, facilidad, alcance y precisión del disparo. Además, el lanzamiento de un dardo mediante este último sistema requiere de un conjunto de movimientos coordinados, complejos $\mathrm{y}$ violentos que pueden asustar a la presa (Raymond, 1986).

Todas estas consideraciones demuestran que el nivel técnico y tecnológico alcanzado en la fabricación de proyectiles líticos durante el Solutrense es suficiente como para que los inicios de la arquería prehistórica puedan situarse en este momento. Lamentablemente, como en muchos problemas planteados en la investigación de las sociedades paleolíticas, la información fragmentaria del registro arqueológico nos impide contrastar fehacientemente las hipótesis elaboradas a partir de la cultura material recuperada de los grupos de cazadoresrecolectores prehistóricos. Como en el caso que nos ocupa, la validación de estas hipótesis se basa en la mayoría de las ocasiones en la viabilidad de las mismas y no en una certeza absoluta corroborada por las evidencias materiales.

Las hipótesis aquí planteadas conforman el punto de partida de un plan de trabajo experimental, en

T. P., 56, n. ${ }^{\circ} 1,1999$ 
curso de realización, sobre distintos sistemas de propulsión, enmangue y efectividad de las puntas ligeras de proyectil del Solutrense Extracantábrico y que pretende lograr un mejor conocimiento de su funcionalidad para corroborar las mismas.

\section{BIBLIOGRAFÍA}

Alrune, F. (1992): "A mesolithic Elm bow approximately 9000 years old". Journal of the Society of Archer-Antiquaries, 35: 47-50.

Allain, J. y Descout, J. (1957): “A propos d'une baguette à rainure armée de silex découverte dans le Magdalénien de Saint-Marcel". L'Anthropologie, 61(5-6): 503512.

Allain, J. y Rigaud, A. (1989): "Colles et mastics au Magdalénien”. En M. Olive et Y. Taborin (eds.): Nature et Fonction des foyers préhistoriques. Actes du Colloque International de Nemours 1987. Mémoires du Musée de Préhistoire d'Ile de France, 2: 221-223. Nemours.

BAYE, J. DE (1974a): "Grottes de Baye. Pointes de flèches en silex à tranchant transversal". Revue Archéologique, XXVII: 401-408.

- (1974b): "Stations de l'époque de la pierre polie dans la vallée du Petit Morin en Champagne". Revue des Sociétés Savantes, VII: 317-319.

BECKER, C.J. (1945): "En 8000-aarigt Stenalderboplads i Holmegaards Mose Fra National museets ArbejdsmarkForelöbelig Meddelelse". Nat. Arb: 61-72.

Bergman, C., McEwen, E. y Miller, R. (1988): "Experimental archery: proyectile velocities and comparison of bow performances". Antiquity, 62: 658-670.

Bocquet, A. (1994): "Charavines il y a 5000 ans". Les Dossiers d'Archeologie, 199: 58-71.

Bourov, G.M. (1973): "Die mesolithischen Kulturen im Aüsserten Europaïschen Nordosten". En S.K. Kozlowski (ed.): The Mesolithic in Europe. Warszawa:129149.

Browne, J. (1938): "Antiquity of the bow". American Antiquity, 3 (4): 358-359.

- (1940): "Projectile points". American Antiquity, 5(3): 209-213.

CAMPS-FABRER, H. (1975): "Tendances actuelles des recherches sur l'industrie de l'os". En H. Camps-Fabrer (org.): Le premier colloque international sur l'indistrie de l'os dans la préhistoire. Abbaye de Sénanque (Vaucluse): 169-173.

Cattelain, P. (1988): Propulseurs. U.I.S.P.P. Commission de nomenclature sur l'industrie de l'os préhistorique. Fiches typologiques de l'industrie osseouse préhistorique. Cahier II. Université de Provence. Marseille.

- (1989): "Un crochet de propulseur solutréen de la grotte de Combe Sauniere I". Bulletin de la Société Préhistorique Française, 86: 213-216.
Clark, J. (1963): "Neolithic bows from Somerset, England, and the prehistory of archery in North-West Europe". Proceedings of the Prehistoric Society, 29: 50-98.

DÉCHELETTE, J. (1908): Manuel d'archéologie préhistorique, celtique et gallo-romaine 1: Archéologie préhistorique. Librairie Alphonse Picard et Fils. Paris. 746 pp.

ELMER, R. (1952): Target archery. Hutchinson. London.

FENEGA, F. (1953): "The weights of chipped stone points: a clue to their functions". Southwestern Journal of Anthropology, 9: 309-323.

ForBIS, R.G. (1960): "The old women's buffalo jump, Alberta Ec-Pl 1". Contributions to Anthropology, 180. Bulletin of National Museum of Canada. Ottawa.

Geneste, J.M. y Plisson, H. (1993): "Hunting technologies and human behavior: lithic analysis of solutrean shouldered points". En H. Kenecht, A. Pike-Tay y R. White (eds.): Before Laxcaux. The complete record of the Early Upper Paleolithic. Boca Ratón, Ann Arbor. London y Tokio: 117-135.

Hamilton, T. (1982): Native american bows. $2^{\mathrm{a}}$ edición. M.A.S.R.S. $1^{\text {a }}$ ed. York, Shumway. 148 pp.

HaRmand, J. (1952): "Remarque sur les bordes abattus: terminologie et usages". Bulletin de la Société Préhistorique Française, XLIX: 554-556.

Heath, E. y Chiara, V. (1977): Brazilian indian archery. Simon Archery Foundation. Manchester.

Hurst, D. (1978): "Arrowheads and atlatl darts: how the stones got the shaft". American Antiquity, 43(3): 462472.

JORDÁ, F. (1958): Avance al estudio de la Cueva de la Lloseta (Ardines, Ribadesella, Asturias). Diputación Provincial de Asturias. Oviedo.

KLOPSTEG, P. (1947): Turkish archery and the composite bow. Klopsteg $2^{\text {nd }}$ edition. Evanston.

Kohl-LaRsen, L. (1958): Wildbeuter im Ostafrica: die Tindiga, eim Jäger und Sammlervolk, Berlin. 165 pp.

LEROI-GourhAN, A. (1983): "Une tête de sagaie à armature de lamelles à dos à Pincevent (S. et M.)". Bulletin de la Société Préhistorique Française, 80(5): 154-156.

Leroi-Gourhan, Arl. y Allain, J. (1979): Lascaux inconnu. $12^{\circ}$ suppl. à Gallia Préhistoire. Paris.

Malmer, M.P. (1968): "Die Mikrolithen in dem Pfeilfund von Loshult". Meddelanden frän Lunds Universitets Historiska Museum: 249-255.

Mathiassen, TH. (1948): Danske Oldsager. IAeldre Stenalder. Köbenhavn. 71 pp.

MAYET, L. y Pissot, J. (1915): Abri sous roche préhistorique de La Colombière près Pocin (Ain). Faculté des Sciences de Lyon. Laboratoire de Géologie et Paléontologie. Section d'Anthropologie et Paléontologie, Lyon. 193 pp.

MuÑoz, F.J. (1997): "La punta de muesca de tipo mediterráneo: análisis tecnológico y funcional". Trabajos de Prehistoria, 54(1): 99-119.

- (e.p.): Las puntas ligeras de proyectil del Solutrense Ex- 
tracantábrico: análisis tecnomorfológico e implicaciones funcionales. Serie Aula Abierta. Universidad Nacional de Educación a Distancia. Madrid.

NutTall, Z. (1891): "The atlatl or spear-thrower of the ancient Mexicans". Papers of the Peabody Museum of American Archaeology and Ethnology, I(3).

ODELL, G.H. y CowAN, F. (1986): "Experiments with spears and arrows on animal targets". Journal of Field Archaeology, 13(2): 195-212.

Olsen, L.D. (1973): Outdoor survival skills, Brigham Young University Press. Utah. 188 pp.

Petterson, M. (1951): "Mikrolithen als Pfeilspitzen". Meddelanden frän Lunds Universitets Historiska Museum: 123-137.

Plisson, H. y Geneste, M. (1989): “Analyse technologique des pointes à cran solutréennes du Placard (Charente), du Fourneau du Diable, du Pech de la Boissiére et de Combe Saunière (Dordogne)". Paléo, 1: 65-106.

Pope, S. (1962): Bows and arrows. Publications in American Archaeology and Ethnology, 13(9). University of California.

- (1974a): Bows and arrows. Reprint of Pope (1923). A study in bows and arrows. University of California Press, Berkeley. 102 pp.

- (1974b): Hunting with the bow and arrow. Popular Lybrary. New York.

RASILlA, M. DE LA (1989): "Reflexiones en torno a la función de los útiles característicos solutrenses". En $\mathrm{Ho}$ menaje a Carlos Cid. Facultad de Geografía e Historia. Universidad de Oviedo. Oviedo: 373-380.

RAYMOND, A. (1986): "Experiments in the function and performances of the weighted atlatl". World Archaeo$\log y, 18(2): 153-176$.

Ripoll, S. (1988): El Solutrense de la Cueva de Ambrosio (Almería, Spain) y su posición cronoestratigráfica en el Mediterráneo Occidental. B.A.R.I.S., 462: 596 pp. ( 2 vols.).

Ripoll, S.; Muñoz, F.J.; Paniagua, J.P.; Calleja, F. y AmaYA, C.:(1997): "La Cueva de Ambrosio (Almería): nuevas aportaciones. El arte rupestre paleolítico”. En J.M. Fullola y N. Soler (eds.): El món mediterrani després del Pleniglacial (18000-12000 B.P.). Banyoles 18-20 mayo de 1995. Centre d'Investigations Arqueològiques. Serie Monogràfica, 17, Girona. 385-398.

Ripoll, S.; PÉrez, S.; LóPez, J.R.; MARTos, J.A. y MuÑIz, M. (1997): "Las estructuras de combustión de la Cueva de Ambrosio. Estudio preliminar". En J.M. ${ }^{\text {a Fullo- }}$ la y N. Soler (eds.): El món mediterrani després del Ple- niglacial (18000-12000 B.P.). Banyoles 18-20 mayo de 1995. Centre d'Investigations Arqueològiques. Serie Monogràfica, 17. Girona: 399-409.

Rozoy, J.G. (1978): Les derniers chasseurs. L'Epipaléolithique en France et en Belgique. 3 vol. Mémoires de la Société Archéologique Champennoise. Charleville. $1.500 \mathrm{pp}$

- (1992a): "Le propulseur et l'arc. Chez les chasseurs préhistoriques. techniques et démographies comparées". Paléo, 4: 175-193.

- (1992b): "Le montage des armatures sur les flèches épipaléolithiques". Revue Archéologique de l'Est, 43(1): 29-38.

- (1993) "Les archers epipaleolithiques: un important progress". Paléo, 5: 263-279.

Rust, A. (1943): Die alt-und mittelrteinzeillichen Funde von Stellmoor. Archaelogisches Institut des deutschen Reiches. Neumünster. $242 \mathrm{pp}$.

Smith, Pн. (1966): Le Solutréen en France. Institut de Géologie du Quaternaire de Bordeaux. Impriméries Delmas. Bordeaux.

STIRLING, M.W. (1960): "The use of the atlatl on Lake Patzcuaro, Michoacan". Smithsonian Institution, Bulletin of Bureau of American Ethnology, 173(57-62): 265268.

STodiek, U. (1993): "Zur technologie der junpaläolithischen speerschleuder". Tübinger Monographien zur Urgeschichte, 9 .

STORDEUR-YEDID, D. (1975): Les aiguilles à chas au Paléolithique. Cahiers du Centre de Recherches Préhistoriques de l'Université de Paris I, Paris.

Tomsen, T. y JesSEn, A. (1904): "Une trouvaille de l' ancien âge de la pierre. La touvaille de Braband". Mémoires de la Société Royale des Antiquaires du Nord. Copenhague: 162-232.

Troels-Smith, J. (1959): “The Muldbjerg dwelling place: An Early Neolithic archaeological site in the Aamosen bog, West-Zealand, Denmark". Smithsonian Report for 1959, publication 4413: 577-601.

- (1961): "Et Pileskaft fra Tidlig Maglemosetid (An arrowshaft from the early Maglemosian)". Aarböger for nordisk Oldkyndighed og historie: 122-146.

VoGT, E. (1952): "Eine mesolithische Harpune aus Schötz (Kt. Luzern). Jahrbuch der Schweizerischen Gesellschaft für Ur-und Frühgeschichte, 42: 155-160.

WyскоFF, D.G. (1964): "The cultural sequence of the Packard Site, Mayes County, Oklahoma”.Archaeological Site Report, 2.

T. P., 56, n. ${ }^{\circ} 1,1999$ 